\title{
ANGULAR MOMENTUM AND MUTUALLY UNBIASED BASES
}

\author{
MAURICE R. KIBLER \\ Institut de physique nucléaire de Lyon \\ IN2P3-CNRS/Université Claude Bernard Lyon 1 \\ 43 bd du 11 novembre 1918 \\ F-69622 Villeurbanne Cedex, France \\ kibler@ipnl.in2p3.fr
}

\begin{abstract}
The Lie algebra of the group $\mathrm{SU}_{2}$ is constructed from two deformed oscillator algebras for which the deformation parameter is a root of unity. This leads to an unusual quantization scheme, the $\left\{J^{2}, U_{r}\right\}$ scheme, an alternative to the familiar $\left\{J^{2}, J_{z}\right\}$ quantization scheme corresponding to common eigenvectors of the Casimir operator $J^{2}$ and the Cartan operator $J_{z}$. A connection is established between the eigenvectors of the complete set of commuting operators $\left\{J^{2}, U_{r}\right\}$ and mutually unbiased bases in spaces of constant angular momentum.
\end{abstract}

Key words: angular momentum; deformations; harmonic oscillator; Lie algebra; polar decomposition; MUBs.

To be published in International Journal of Modern Physics B 


\section{Introduction}

In recent years, the notion of deformed oscillator algebra and its extension to deformed Lie algebra, or Hopf algebra in mathematical parlance, ${ }^{1-5}$ proved to be useful in various fields of theoretical physics. For instance, one- and two-parameter deformations of oscillator algebras and Lie algebras were successfully applied to statistical mechanics ${ }^{6-13}$ and to nuclear, atomic and molecular physics. ${ }^{14-18}$ In the case where the deformation parameter is a root of unity, let us also mention the importance of deformed oscillator algebras for the definition of $k$-fermions, which are objects interpolating between fermions and bosons, ${ }^{19}$ and the study of fractional supersymmetry. ${ }^{20}$

The aim of this note is two-fold. First, we show how a deformation of two truncated harmonic oscillators leads to a polar decomposition of the Lie algebra of $\mathrm{SU}_{2}$. Such a decomposition is especially appropriate for developing the representation theory and the Wigner-Racah algebra of $\mathrm{SU}_{2}$ in a non-standard basis adapted to cyclical symmetry. ${ }^{21}$ Second, we establish a contact between the corresponding bases for spaces of constant angular momentum and the so-called mutually unbiased bases (MUBs) in a finite-dimensional Hilbert space. The latter bases ${ }^{22-44}$ play a central role in quantum information theory. In particular, the use of quantum-mechanical states belonging to MUBs is of paramount importance in quantum cryptography (securing quantum key exchange) and quantum state tomography (deciphering a quantum state).

\section{Angular Momentum Theory in a Nonstandard Basis}

\subsection{The Lie algebra of $\mathrm{SU}_{2}$ from two oscillator algebras}

Let $\mathcal{F}(1)$ and $\mathcal{F}(2)$ be two finite-dimensional Hilbert spaces of dimension $k$ with $k \in$ $\mathbf{N} \backslash\{0,1\}$. We use $(\mid)$ to denote the inner product on $\mathcal{F}(i)$ and, for each space $\mathcal{F}(i)$ with $i=1,2$, we choose an orthonormal basis $\left.\left\{\mid n_{i}\right): n_{i}=0,1, \cdots, k-1\right\}$. Let $\left(a_{i-}, a_{i+}, N_{i}\right)$ be a triplet of linear operators on $\mathcal{F}(i)$ defined by

$$
\begin{gathered}
\left.\left.a_{i \pm} \mid n_{i}\right)=\left(\left[n_{i}+s \pm \frac{1}{2}\right]_{q}\right)^{\alpha_{i \pm}} \mid n_{i} \pm 1\right) \\
\left.\left.\left.\left.a_{i+} \mid k-1\right)=0, \quad a_{i-} \mid 0\right)=0, \quad N_{i} \mid n_{i}\right)=n_{i} \mid n_{i}\right)
\end{gathered}
$$

where

$$
s=\frac{1}{2}, \quad \alpha_{i \pm}=\frac{1 \pm(-1)^{i}}{2}, \quad q=\exp \left(\frac{2 \pi \mathrm{i}}{k}\right), \quad[x]_{q}=\frac{1-q^{x}}{1-q}, \quad x \in \mathbf{R}
$$

with $i=1,2$. It can be shown that the operators $a_{i-}, a_{i+}$ and $N_{i}$ satisfy the following relations

$$
a_{i-} a_{i+}-q a_{i+} a_{i-}=1, \quad\left(a_{i \pm}\right)^{k}=0, \quad\left[N_{i}, a_{i \pm}\right]= \pm a_{i \pm}, \quad N_{i}^{\dagger}=N_{i}
$$

where we use the notation $A^{\dagger}$ for the adjoint of $A$ and $[A, B]$ for the commutator of the operators $A$ and $B$. The two algebras defined by Eq. (1) with $i=1,2$ are two commuting 
oscillator algebras with $q$ being a root of unity; this is reminiscent of the two oscillator algebras used for the introduction of $k$-fermions. ${ }^{19,20}$

We now consider the space $\mathcal{F}_{k}=\mathcal{F}(1) \otimes \mathcal{F}(2)$ of dimension $k^{2}$. An orthonormal basis for $\mathcal{F}_{k}$ is provided by the vectors

$$
\left.\left.\left.\mid n_{1}, n_{2}\right)=\mid n_{1}\right) \otimes \mid n_{2}\right), \quad n_{i}=0,1, \cdots, k-1, \quad i=1,2
$$

The key of our derivation of a nonstandard basis of $\mathrm{SU}_{2}$ consists in defining the two linear operators

$$
H=\sqrt{N_{1}\left(N_{2}+1\right)}, \quad U_{r}=s_{1+} s_{2-}
$$

where

$$
s_{i \pm}=a_{i \pm}+\mathrm{e}^{\frac{1}{2} \mathrm{i} \phi_{r}} \frac{1}{[k-1]_{q} !}\left(a_{i \mp}\right)^{k-1}
$$

for $i=1,2$. In the operator $s_{i \pm}$, the phase $\phi_{r}$ is an arbitrary real parameter taken in the form

$$
\phi_{r}=\pi(k-1) r, \quad r \in \mathbf{R}
$$

and $[n]_{q}$ ! stands for the $q$-deformed factorial defined by

$$
\forall n \in \mathbf{N}^{*}:[n]_{q} !=[1]_{q}[2]_{q} \cdots[n]_{q}, \quad[0]_{q} !=1
$$

It is immediate to show that the action of $H$ and $U_{r}$ on $\mathcal{F}_{k}$ is given by

$$
\left.\left.H \mid n_{1}, n_{2}\right)=\sqrt{n_{1}\left(n_{2}+1\right)} \mid n_{1}, n_{2}\right), \quad n_{i}=0,1,2, \cdots, k-1, \quad i=1,2
$$

and

$$
\begin{gathered}
\left.\left.U_{r} \mid n_{1}, n_{2}\right)=\mid n_{1}+1, n_{2}-1\right), \quad n_{1} \neq k-1, \quad n_{2} \neq 0 \\
\left.\left.U_{r} \mid k-1, n_{2}\right)=\mathrm{e}^{\frac{1}{2} \mathrm{i} \phi_{r}} \mid 0, n_{2}-1\right), \quad n_{2} \neq 0 \\
\left.\left.U_{r} \mid n_{1}, 0\right)=\mathrm{e}^{\frac{1}{2} \mathrm{i} \phi_{r}} \mid n_{1}+1, k-1\right), \quad n_{1} \neq k-1 \\
\left.\left.U_{r} \mid k-1,0\right)=\mathrm{e}^{\mathrm{i} \phi_{r}} \mid 0, k-1\right)
\end{gathered}
$$

The operators $H$ and $U_{r}$ satisfy interesting properties. The operator $H$ is Hermitean and the operator $U_{r}$ is unitary. Furthermore, the action of $U_{r}$ on the space $\mathcal{F}_{k}$ is cyclic in the sense that

$$
\left(U_{r}\right)^{k}=\mathrm{e}^{\mathrm{i} \phi_{r}} I
$$

where $I$ is the identity operator. 
From the Schwinger work on angular momentum, ${ }^{45}$ we introduce

$$
J=\frac{1}{2}\left(n_{1}+n_{2}\right), \quad M=\frac{1}{2}\left(n_{1}-n_{2}\right)
$$

We shall use the notation

$$
\left.|J M\rangle \equiv \mid J+M, J-M)=\mid n_{1}, n_{2}\right)
$$

For a fixed value of $J$, the label $M$ can take $2 J+1$ values $M=-J,-J+1, \cdots, J$. For fixed $k$, the maximum value of $J$ is $J=J_{\max }=k-1$ and the following value of $J$

$$
J=j=\frac{1}{2}(k-1)
$$

is admissible. For a given value of $k \in \mathbf{N} \backslash\{0,1\}$, the $2 j+1=k$ vectors $|j m\rangle$ belong to the vector space $\mathcal{F}_{k}$. Let $\varepsilon(j)$ be the subspace of $\mathcal{F}_{k}$, of dimension $\operatorname{dim} \varepsilon(j)=k$, spanned by the $k$ vectors $|j m\rangle$ with $m=-j,-j+1, \cdots, j$. We can thus associate the space $\varepsilon(j)$ for $j=\frac{1}{2}, 1, \frac{3}{2}, \cdots$ to the values $k=2,3,4, \cdots$, respectively. The subspace $\varepsilon(j)$ of $\mathcal{F}_{k}$ is stable under $H$ and $U_{r}$. Indeed, the action of the operators $H$ and $U_{r}$ on the space $\varepsilon(j)$ can be described by

$$
H|j m\rangle=\sqrt{(j+m)(j-m+1)}|j m\rangle
$$

and

$$
U_{r}|j m\rangle=[1-\delta(m, j)]|j m+1\rangle+\delta(m, j) \mathrm{e}^{\mathrm{i} \phi_{r}}|j-j\rangle
$$

We can check that the operator $H$ is Hermitean and the operator $U_{r}$ is unitary on the space $\varepsilon(j)$. Furthermore, we have $\left(U_{r}\right)^{2 j+1}=\mathrm{e}^{\mathrm{i} \phi_{r}} I$ which reflects the cyclical character of $U_{r}$ on $\varepsilon(j)$.

We are now in a position to give a realization of the Lie algebra of the group $\mathrm{SU}_{2}$ in terms of $U_{r}, N_{1}$ and $N_{2}$. Let us define the three operators

$$
J_{+}=H U_{r}, \quad J_{-}=U_{r}^{\dagger} H, \quad J_{z}=\frac{1}{2}\left(N_{1}-N_{2}\right)
$$

It is straightforward to check that

$$
J_{ \pm}|j m\rangle=\sqrt{(j \mp m)(j \pm m+1)}|j m \pm 1\rangle, \quad J_{z}|j m\rangle=m|j m\rangle
$$

Consequently, we get the commutation relations

$$
\left[J_{z}, J_{ \pm}\right]= \pm J_{ \pm}, \quad\left[J_{+}, J_{-}\right]=2 J_{z}
$$

which correspond to the Lie algebra of $\mathrm{SU}_{2}$. 


\subsection{An nonstandard basis for the group $\mathrm{SU}_{2}$}

The decomposition of the shift operators $J_{+}$and $J_{-}$in terms of $H$ and $U_{r}$ coincides with the polar decomposition worked out in Refs. 46 and 47 in a completely different way. This is easily seen by taking the matrix elements of $U_{r}$ and $H$ in the $\left\{J^{2}, J_{z}\right\}$ quantization scheme and by comparing these elements to the ones of the operators $\Upsilon$ and $J_{T}$ in Ref. 46. We are thus left with $H=J_{T}$ and, by identifying the arbitrary phase $\varphi$ of Ref. 46 with $\phi_{r}$, we obtain $U_{r}=\Upsilon$ so that $J_{+}=J_{T} \Upsilon$ and $J_{-}=\Upsilon^{\dagger} J_{T}$.

It is immediate to check that the Casimir operator $J^{2}$ of the Lie algebra $\mathrm{su}_{2}$ can be rewritten as

$$
J^{2}=\frac{1}{4}\left(N_{1}+N_{2}\right)\left(N_{1}+N_{2}+2\right)
$$

in terms of $N_{1}$ and $N_{2}$. It is a simple matter of calculation to prove that $J^{2}$ commutes with $U_{r}$ for any value of $r$. Therefore, for $r$ fixed, the commuting set $\left\{J^{2}, U_{r}\right\}$ provides us with an alternative to the familiar commuting set $\left\{J^{2}, J_{z}\right\}$ of angular momentum theory.

The eigenvalues and the common eigenvectors of the complete set of commuting operators $\left\{J^{2}, U_{r}\right\}$ can be easily found. This leads to the following result.

Result 1. The spectra of the operators $U_{r}$ and $J^{2}$ are given by

$$
U_{r}\left|j n_{\alpha} ; r\right\rangle=q^{-\alpha}\left|j n_{\alpha} ; r\right\rangle, \quad J^{2}\left|j n_{\alpha} ; r\right\rangle=j(j+1)\left|j n_{\alpha} ; r\right\rangle
$$

where

$$
\left|j n_{\alpha} ; r\right\rangle=\frac{1}{\sqrt{2 j+1}} \sum_{m=-j}^{j} q^{\alpha m}|j m\rangle, \quad q=\exp \left(\mathrm{i} \frac{2 \pi}{2 j+1}\right)
$$

with the range of values

$$
\alpha=-j r+n_{\alpha}, \quad n_{\alpha}=0,1, \cdots, 2 j
$$

where $2 j \in \mathbf{N}^{*}$ and $r \in \mathbf{R}$.

Each vector $\left|j n_{\alpha} ; r\right\rangle$ can be considered as a discrete Fourier transform ${ }^{47}$ in the finitedimensional Hilbert space $\varepsilon(j)$. As a matter of fact, the inter-basis expansion coefficients

$$
\left\langle j m \mid j n_{\alpha} ; r\right\rangle=\frac{1}{\sqrt{2 j+1}} \exp \left[\mathrm{i} \frac{2 \pi}{2 j+1}\left(-j r+n_{\alpha}\right) m\right]
$$

(with $m=-j,-j+1, \cdots, j$ and $n_{\alpha}=0,1, \cdots, 2 j$ ) in Eq. (2) define a unitary transformation, in $\varepsilon(j)$ (with $j=\frac{1}{2}, 1, \frac{3}{2}, \cdots$ ), that allows to pass from the well-known orthonormal standard spherical basis

$$
s(j)=\{|j m\rangle: m=-j,-j+1, \cdots, j\}
$$

to the orthonormal non-standard basis

$$
b_{r}(j)=\left\{\left|j n_{\alpha} ; r\right\rangle: n_{\alpha}=0,1, \cdots, 2 j\right\}
$$


For a given value of $r$, the basis $b_{r}(j)$ is an alternative to the spherical basis $s(j)$ of the space $\varepsilon(j)$. Two bases $b_{r}(j)$ and $b_{s}(j)$ with $r \neq s$ are thus two equally admissible orthonormal bases for $\varepsilon(j)$. The state vectors of the bases $b_{r}(j)$ and $b_{s}(j)$ are common eigenstates of $\left\{J^{2}, U_{r}\right\}$ and $\left\{J^{2}, U_{s}\right\}$, respectively. The overlap between the bases $b_{r}(j)$ and $b_{s}(j)$ is controlled by

$$
\left\langle j n_{\alpha} ; r \mid j n_{\beta} ; s\right\rangle=\frac{1}{2 j+1} \frac{\sin (\alpha-\beta) \pi}{\sin (\alpha-\beta) \frac{\pi}{2 j+1}}
$$

with $\alpha=-j r+n_{\alpha}$ and $\beta=-j s+n_{\beta}$ where $n_{\alpha}, n_{\beta}=0,1, \cdots, 2 j$.

\section{Connection with Mutually Unbiased Bases}

We are now ready for establishing contact with MUBs. Let $\varepsilon(d)$ be a Hilbert space of dimension $d$ endowed with the inner product $\langle\mid\rangle$. Two orthonormal bases $A=\{|A \alpha\rangle$ : $\alpha=0,1, \cdots, d-1\}$ and $B=\{|B \beta\rangle: \beta=0,1, \cdots, d-1\}$ are said to be mutually unbiased if and only if $|\langle A \alpha \mid B \beta\rangle|=\frac{1}{\sqrt{d}}$ for all $\alpha \in\{0,1, \cdots, d-1\}$ and all $\beta \in$ $\{0,1, \cdots, d-1\}$. For an arbitrary value of $d$, the number of MUBs cannot be greater than $d+1 .^{22-25}$

We note in passing that the latter result can be justified from group theory. The $d$ orthonormal vectors of a basis for $\varepsilon(d)$ can be considered as a basis for a fundamental representation of dimension $d$ of the group $\mathrm{SU}_{d}$. This group is of dimension $d^{2}-1$ and of rank (i.e., the number of Cartan generators) $d-1$. Therefore, the maximal number of independent sets of $d-1$ commuting operators it is possible to construct from the $d^{2}-1$ generators of $\mathrm{SU}_{d}$ is $\frac{d^{2}-1}{d-1}=d+1$. This is precisely the maximum number of MUBs for the space $\varepsilon(d)$. Indeed, the limit $d+1$ is reached if $d$ is a prime number ${ }^{23}$ or a power of a prime number. ${ }^{24-30}$

It is also interesting to note that a connection exists between MUBs and various geometries (e.g., see Refs. 31, 36, 38, 40 and 43). In particular, according to the SPR conjecture ${ }^{31}$ for $d$ fixed with $d$ not equal to a power of a prime number, the problem of the existence of a complete set of $d+1$ MUBs would be equivalent to the one of the existence of projective planes of order $d$.

We derive below some preliminary results of interest for an investigation of a relation between the $\left\{J^{2}, U_{r}\right\}$ scheme and MUBs. To begin with, from Eq. (2), we have the following result.

Result 2. The overlap between the bases $s(j)$ and $b_{r}(j)$ satifies

$$
\left|\left\langle j m \mid j n_{\alpha} ; r\right\rangle\right|^{2}=\frac{1}{\operatorname{dim} \varepsilon(j)}
$$

so that $s(j)$ and $b_{r}(j)$ are two MUBs for the space $\varepsilon(j)$.

As an illustration, we consider the space $\varepsilon\left(\frac{1}{2}\right)$ of dimension 2. Equation (2) yields

$$
\left|\frac{1}{2} 0 ; 0\right\rangle=\frac{1}{\sqrt{2}}\left(\left|\frac{1}{2}-\frac{1}{2}\right\rangle+\left|\frac{1}{2} \frac{1}{2}\right\rangle\right), \quad\left|\frac{1}{2} 1 ; 0\right\rangle=\frac{\mathrm{i}}{\sqrt{2}}\left(-\left|\frac{1}{2}-\frac{1}{2}\right\rangle+\left|\frac{1}{2} \frac{1}{2}\right\rangle\right)
$$


for $r=0$ and

$$
\left|\frac{1}{2} 0 ; 1\right\rangle=\frac{1}{\sqrt{2}}\left(\rho\left|\frac{1}{2}-\frac{1}{2}\right\rangle+\rho^{-1}\left|\frac{1}{2} \frac{1}{2}\right\rangle\right), \quad\left|\frac{1}{2} 1 ; 1\right\rangle=\frac{1}{\sqrt{2}}\left(\rho^{-1}\left|\frac{1}{2}-\frac{1}{2}\right\rangle+\rho\left|\frac{1}{2} \frac{1}{2}\right\rangle\right)
$$

for $r=1$ with $\rho=\mathrm{e}^{\mathrm{i} \frac{\pi}{4}}$. It is evident that the three bases $s\left(\frac{1}{2}\right), b_{0}\left(\frac{1}{2}\right)$ and $b_{1}\left(\frac{1}{2}\right)$ constitute a complete set of MUBs for $\varepsilon\left(\frac{1}{2}\right)$.

The situation is not so simple for $2 j \in \mathbf{N} \backslash\{0,1\}$. For fixed $j$, the eigenfunctions of the operators $U_{r}$ and $U_{s}$, with $r \neq s$, are not necessarily independent. We give in what follows some results that can be useful for $2 j \neq 1$.

Result 3. By assuming

$$
s=r+\frac{n_{\beta}-n_{\alpha}}{j}+\frac{2 j+1}{j} k_{\alpha \beta}, \quad k_{\alpha \beta} \in \mathbf{Z}
$$

we get

$$
\left|j n_{\beta} ; s\right\rangle=(-1)^{2 j k_{\alpha \beta}}\left|j n_{\alpha} ; r\right\rangle
$$

and the corresponding bases $b_{r}(j)$ and $b_{s}(j)$ are not MUBs.

Result 4. The commutator of $U_{s}$ and $U_{r}$ on $\varepsilon(j)$ assumes the form

$$
\left[U_{s}, U_{r}\right]=\left(\mathrm{e}^{\mathrm{i} \phi_{s}}-\mathrm{e}^{\mathrm{i} \phi_{r}}\right)[|j,-j\rangle\langle j, j-1|-| j,-j+1\rangle\langle j, j|]
$$

Therefore, a necessary and sufficient condition that the operators $U_{s}$ and $U_{r}$ commute is

$$
s=r+\frac{x}{j}, \quad x \in \mathbf{Z}
$$

We note that Eq. (3) implies Eq. (4).

Result 5. On the space $\varepsilon(j)$, let $Z$ be the familiar phase operator defined by

$$
\forall m \in\{-j,-j+1, \cdots, j\}: Z|j m\rangle=q^{-m}|j m\rangle
$$

and, for fixed $r$, let $V_{r a}$ be the $2 j+1$ unitary operators given by

$$
V_{r a}=U_{r} Z^{a}=q^{a} Z^{a} U_{r}, \quad a=0,1, \cdots, 2 j
$$

(cf. the Weyl commutation relation rule). The Hilbert-Schmidt inner product of the operators $V_{s b}$ and $V_{r a}$ is

$$
\operatorname{tr}\left(V_{s b}^{\dagger} V_{r a}\right)=(2 j+1) \delta(a, b)+q^{j(b-a)}\left[\mathrm{e}^{\mathrm{i}\left(\phi_{r}-\phi_{s}\right)}-1\right]
$$

where the trace is taken on $\varepsilon(j)$ and where $r \in \mathbf{R}, s \in \mathbf{R}$ and $a, b=0,1, \cdots, 2 j$. For $r$ and $s$ such that the condition (4) is satisfied, we have

$$
\operatorname{tr}\left(V_{s b}^{\dagger} V_{r a}\right)=(2 j+1) \delta(a, b)
$$


with $r \in \mathbf{R}, s \in \mathbf{R}$ and $a, b=0,1, \cdots, 2 j$.

We note that $2 j=1$ is the sole case for which it is possible to find $r$ and $s$ such that $\operatorname{tr}\left(U_{s}^{\dagger} U_{r}\right)=0$. This explains the peculiarity of the case $2 j=1$.

Result 6. In the case where $2 j+1$ is prime, following the works in Refs. 26, 27, 35 and 47 , for a given value of $r$ let $M$ be the set of unitary operators

$$
M=\left\{V_{r a}: a=0,1, \cdots, 2 j\right\}
$$

generated by the two generalized Weyl-Pauli operators $U_{r}$ and $Z$. The vectors of the spherical basis $s(j)$ and the eigenvectors of the $2 j+1$ operators in $M$ provide a set of of $2 j+2$ MUBs for the Hilbert space $\varepsilon(j)$ of dimension $2 j+1$.

The derivation of the latter result easily follows by adapting the proof of Theorem 2.3 of Ref. 26.

As an example, we treat the case $j=1$ with $r=0$ for which the 12 vectors of the 4 MUBs can be described by a single simple formula. The $2 j+2=4$ MUBs consist of the spherical basis $s(1)$ and of the 3 bases (corresponding to $a=0,1$ and 2) spanned by the vectors

$$
\Psi_{a}\left(n_{\alpha}\right)=\frac{1}{\sqrt{3}}\left(\omega^{-n_{\alpha}+a}|1-1\rangle+|10\rangle+\omega^{n_{\alpha}+a}|11\rangle\right)
$$

with $n_{\alpha}=0,1,2$ and $a=0,1,2$ (as usual, $\omega=\mathrm{e}^{\mathrm{i} \frac{2 \pi}{3}}$ ). The vectors $|1 m\rangle$ of the spherical basis $s(1)$ are eigenvectors of $J_{z}$ with the real eigenvalues $m$. The case $a=0$ corresponds to the basis $b_{0}(1)$, the vectors $\left|1 n_{\alpha} ; 0\right\rangle$ of which are eigenvectors of $V_{00}=U_{0}$ with the complex eigenvalues $\omega^{-n_{\alpha}}$. More generally, for fixed $a$ (with $a=0,1$ or 2), the vectors of the basis $\left\{\Psi_{a}\left(n_{\alpha}\right): n_{\alpha}=0,1\right.$ and 2$\}$ are eigenvectors of the operators $V_{0 a}$ :

$$
V_{0 a} \Psi_{a}\left(n_{\alpha}\right)=\omega^{-n_{\alpha}-a} \Psi_{a}\left(n_{\alpha}\right)
$$

As a résumé, by introducing the notation

$$
N_{x y z}(x, y, z) \equiv N_{x y z}(x|1-1\rangle+y|10\rangle+z|11\rangle), \quad N_{x y z}=\frac{1}{\sqrt{|x|^{2}+|y|^{2}+|z|^{2}}}
$$

we have the 4 MUBs

$$
\begin{aligned}
& s(1):(1,0,0) ;(0,1,0) ;(0,0,1) \\
& a=0 \quad: \quad \frac{1}{\sqrt{3}}(1,1,1) ; \frac{1}{\sqrt{3}}\left(\omega^{2}, 1, \omega\right) ; \frac{1}{\sqrt{3}}\left(\omega, 1, \omega^{2}\right) \\
& a=1 \quad: \quad \frac{1}{\sqrt{3}}(\omega, 1, \omega) ; \frac{1}{\sqrt{3}}\left(1,1, \omega^{2}\right) ; \frac{1}{\sqrt{3}}\left(\omega^{2}, 1,1\right) \\
& a=2 \quad: \quad \frac{1}{\sqrt{3}}\left(\omega^{2}, 1, \omega^{2}\right) ; \frac{1}{\sqrt{3}}(\omega, 1,1) ; \frac{1}{\sqrt{3}}(1,1, \omega)
\end{aligned}
$$

for the space $\varepsilon(1)$. Note that, for fixed $a$ (with $a=0,1$ or 2 ), the basis $\left\{\Psi_{a}\left(n_{\alpha}\right): n_{\alpha}=\right.$ $0,1$ and 2$\}$ spans the regular representation of the cyclic group $Z_{3}$. Note also that, the basis $b_{0}(1)$ corresponds to the three irreducible vector representations of $Z_{3}$ while the bases for $a=1$ and $a=2$ correspond to irreducible projective representations of $Z_{3}$. 


\section{Concluding Remarks}

The derivation of the usual (i.e., non-deformed) Lie algebra $\mathrm{su}_{2}$ was achieved in Sec. 2 by adapting the Schwinger trick ${ }^{45,47}$ to the case of two deformed oscillator algebras corresponding to a coupled pair of truncated harmonic oscillators. This constitutes an unsual result for Lie algebras. In the context of deformations, we generally start from a Lie algebra, then deform it and finally find a realization in terms of deformed oscillator algebras. Here we started from two $q$-deformed oscillator algebras from which we derived the non-deformed Lie algebra $\mathrm{su}_{2}$.

The polar decomposition of the ladder operators of $\mathrm{su}_{2}$ inherent to our derivation of $\mathrm{su}_{2}$ led to the scheme $\left\{J^{2}, U_{r}\right\}$, an alternative to the standard scheme $\left\{J^{2}, J_{z}\right\}$ of angular momentum theory, a theory familiar to the physicist.

Some of the known results about MUB's were explored in Sec. 3 in the framework of angular momentum theory with a special emphasis on the unitay operator $U_{r}$. This shows that the idea of deformations (and possibly Hopf algebras), especially for a deformation parameter taken as a root of unity, could be useful for investigating MUBs. It is also hoped that the so-called Wigner-Racah unit tensors ${ }^{45}$ acting on a subspace of constant angular momentum $\varepsilon(j)$ and spanning the Lie algebra of the unitary group $\mathrm{U}_{2 j+1}$ might be useful for characterizing the operators $V_{r a}$ of Sec. 3. Furthermore, it is worth noting that the parameter $r$ in $V_{r a}$ introduces a further degree of freedom. In this respect, let us mention that, when $2 j+1$ is an odd prime number, by replacing $r$ in Eq. (2) by the $m$-dependent parameter

$$
r(m)=-a \frac{(j+m)^{2}}{j m}, \quad m \neq 0, \quad a=0,1, \ldots, 2 j
$$

we generate, together with the spherical basis $s(j), 2 j+2$ MUBs for the space $\varepsilon(j)$. This amounts in last analysis to redefining the operator $U_{r}$.

These matters deserve to be further worked out and should be the object of a future work.

\section{Acknowledgements}

The author is grateful to Michel Planat for very interesting correspondence (and the suggestion of using Galois fields and Galois rings for classifiyng particles and chemical elements).

\section{References}

[1] M. Arik and D.D. Coon, J. Math. Phys. 17, 524 (1976).

[2] L.C. Biedenharn, J. Phys. A22, L873 (1989).

[3] C.-P. Sun and H.-C. Fu, J. Phys. A22, L983 (1989).

[4] A.J. Macfarlane, J. Phys. A22, 4581 (1989). 
[5] M.R. Kibler, in Symmetry and Structural Properties of Condensed Matter, Eds. W. Florek, D. Lipiński and T. Lulek (World Scientific, Singapore, 1993).

[6] M.A. Martín-Delgado, J. Phys. A24, L1285 (1991).

[7] G. Su and M.-1. Ge, Phys. Lett. A173, 17 (1993).

[8] J.A. Tuszyński, J.L. Rubin, J. Meyer and M. Kibler, Phys. Lett. A175, 173 (1993).

[9] V.I. Man'ko, G. Marmo, S. Solimeno and F. Zaccaria, Phys. Lett. A176, 173 (1993).

[10] R.-R. Hsu and C.-R. Lee, Phys. Lett. A180, 314 (1993).

[11] R.K. Gupta, C.T. Bach and H. Rosu, J. Phys. A27, 1427 (1994).

[12] R.-S. Gong, Phys. Lett. A199, 81 (1995).

[13] M. Daoud and M. Kibler, Phys. Lett. A206, 13 (1995).

[14] S. Iwao, Prog. Theor. Phys. 83, 363 (1990).

[15] D. Bonatsos, P.P. Raychev, R.P. Roussev and Yu.F. Smirnov, Chem. Phys. Lett. 175, 300 (1990). D. Bonatsos, S.B. Drenska, P.P. Raychev, R.P. Roussev and Yu.F. Smirnov, J. Phys. G17, L67 (1991). D. Bonatsos, P.P. Raychev and A. Faessler, Chem. Phys. Lett. 178, 221 (1991). D. Bonatsos, E.N. Argyres and P.P. Raychev, J. Phys. A24, L403 (1991).

[16] M. Kibler and T. Négadi, J. Phys. A24, 5283 (1991). T. Négadi and M. Kibler, J. Phys. A25, L157 (1992).

[17] Z. Chang and H. Yan, Phys. Lett. A154, 254 (1991). Z. Chang H.-Y. Guo and H. Yan, Phys. Lett. A156, 192 (1991). Z. Chang and H. Yan, Phys. Lett. A158, 242 (1991).

[18] L. Jenkovszky, M. Kibler and A. Mishchenko, Mod. Phys. Lett. A10, 51 (1995). R. Barbier and M. Kibler, in Finite Dimensional Integrable Systems, Eds. A.N. Sissakian and G.S. Pogosyan (JINR, Dubna, 1995). R. Barbier and M. Kibler, in Modern Group Theoretical Methods in Physics, Eds. J. Bertrand, M. Flato, J.-P. Gazeau, D. Sternheimer and M. Irac-Astaud (Kluwer, Dordrecht, 1995). R. Barbier and M. Kibler, Rep. Math. Phys. 38, 221 (1996).

[19] M. Daoud, Y. Hassouni and M. Kibler, in Symmetries in Science X, Eds. B. Gruber and M. Ramek (Plenum Press, New York, 1998). M. Daoud, Y. Hassouni and M. Kibler, Phys. Atom. Nuclei 61, 1821 (1998).

[20] M. Daoud and M. Kibler, Int. J. Quantum Chem. 91, 551 (2003). M.R. Kibler and M. Daoud, in: Fundamental world of quantum chemistry, Vol. III, Eds. E.J. Brändas and E.S. Kryachko (Kluwer, Dordrecht, 2004). M. Daoud and M. Kibler, Phys. Lett. A321, 147 (2004); Phys. Atom. Nuclei 68, 1654 (2005).

[21] M.R. Kibler, in: Symmetry and Structural Properties of Condensed Matter, Eds. T. Lulek, B. Lulek and A. Wal (World Scientific, Singapore, 1999). M. Kibler and M. Daoud, Recent Res. Devel. Quantum. Chem. 2, 91 (2001). M.R. Kibler, Collect. Czech. Chem. Commun. 70, 771 (2005). 
[22] P. Delsarte, J.M. Goethals and J.J. Seidel, Philips Res. Repts. 30, 91 (1975).

[23] I.D. Ivanović, J. Phys. A14, 3241 (1981).

[24] W.K. Wootters and B.D. Fields, Ann. Phys. 191, 363 (1989).

[25] A.R. Calderbank, P.J. Cameron, W.M. Kantor and J.J. Seidel, Proc. London Math. Soc. 75, 436 (1997).

[26] S. Bandyopadhyay, P.O. Boykin, V. Roychowdhury and F. Vatan, Algorithmica 34, 512 (2002).

[27] J. Lawrence, Č. Brukner and A. Zeilinger, Phys. Rev. A65, 032320 (2002).

[28] S. Chaturvedi, Phys. Rev. A65, 044301 (2002).

[29] A.O. Pittenger and M.H. Rubin, Linear Alg. Appl. 390, 255 (2004); J. Phys. A38, 6005 (2005).

[30] A. Klappenecker and M. Rötteler, Lecture Notes in Computer Science 2948, 137 (2004).

[31] M. Saniga, M. Planat and H. Rosu, J. Opt. B: Quantum Semiclass. Opt. 6, L19 (2004).

[32] K.S. Gibbons, M.J. Hoffman and W.K. Wootters, Phys. Rev. A70, 062101 (2004).

[33] T. Durt, J. Phys. A38, 5267 (2005).

[34] P. Wocjan and T. Beth, Quant. Inf. and Comp. 5, 93 (2005).

[35] A.B. Klimov, L.L. Sánchez-Soto and H. de Guise, J. Phys. A38, 2747 (2005).

[36] M. Saniga and M. Planat, Chaos, Solitons and Fractals 26, 1267 (2005).

[37] M. Planat and H. Rosu, Eur. Phys. J. D36, 133 (2005).

[38] W.K. Wootters, Found. Phys. (to appear).

[39] C. Archer, quant-ph/0312204

[40] I. Bengtsson, quant-ph/0406174. I. Bengtsson and Å. Ericsson, quant-ph/0410120.

[41] M. Grassl, quant-ph/0406175.

[42] M. Planat, H. Rosu, S. Perrine and M. Saniga, quant-ph/0409081

[43] M. Saniga and M. Planat, math-ph/0506057

[44] P. O. Boykin, M. Sitharam, P. H. Tiep and P. Wocjan, quant-ph/0506089

[45] J. Schwinger, in Quantum Theory of Angular Momemtum, Eds. L.C. Biedenharn and H. van Dam (Academic Press, New York, 1965). See also: M. Kibler and G. Grenet, J. Math. Phys. 21, 422 (1980). 
[46] J.-M. Lévy-Leblond, Rev. Mex. Física 22, 15 (1973).

[47] A. Vourdas, Rep. Prog. Phys. 67, 267 (2004). See also: A. Vourdas, Phys. Rev. A41, 1653 (1990); J. Phys. A29, 4275 (1996). 